Handbuch Ethik 


\section{Handbuch Ethik}

Herausgegeben von

Marcus Düwell, Christoph Hübenthal

und Micha $\mathrm{H}$. Werner

Verlag J. B. Metzler

Stuttgart · Weimar 
Die Deutsche Bibliothek - Cip-Einheitsaufnahme

Handbuch Ethik / hrsg. von Marcus Düwell ...

- Stuttgart ; Weimar : Metzler, 2002

ISBN 978-3-476-01749-9

ISBN 978-3-476-01749-9

ISBN 978-3-476-02713-9 (eBook)

DOI 10.1007/978-3-476-02713-9

Dieses Werk einschließlich aller seiner Teile ist urheberrechtlich geschützt. Jede Verwertung außerhalb der engen Grenzen des Urheberrechtsgesetzes ist ohne Zustimmung des Verlages unzulässig und strafbar. Das gilt insbesondere für Vervielfältigungen, Übersetzungen, Mikroverfilmungen und die Einspeicherung und Verarbeitung in elektronischen Systemen.

(C) 2002 Springer-Verlag GmbH Deutschland Ursprünglich erschienen bei J. B. Metzlersche Verlagsbuchhandlung und Carl Ernst Poeschel Verlag GmbH in Stuttgart 2002

www.metzlerverlag.de

info@metzlerverlag.de 


\section{Inhalt}

Vorwort $\ldots \ldots \ldots \ldots \ldots \ldots$. . . . . .

\section{Einleitung}

II. Ethische Theorien im Überblick . . 25

A. Metaethik und deskriptive Ethik . . . . . . 25

1. Metaethik - ein systematischer Überblick . . 25

2. Kognitivismus / Nonkognitivismus . . . . . 36

3. Realismus / Intuitionismus / Naturalismus . 49

B. Ansätze Normativer Ethik . . . . . . . . . . 61

B.1 Teleologische Ansätze . . . . . . . . 61

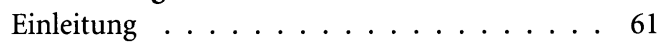

4. Aristoteles . . . . . . . . . . . . 69

5. Eudaimonismus . . . . . . . . . . . 82

6. Utilitarismus / Ethischer Egoismus . . . . . . 95

7. Wertethik . . . . . . . . . . 108

B.2 Deontologische Ansätze . . . . . . . . 122

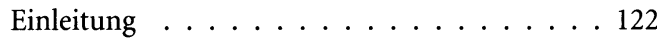

8. Kant . . . . . . . . . . . 128

9. Diskursethik . . . . . . . . . . . . 140

10. Handlungsreflexive Moralbegründung . . . 152

11. Kontraktualismus . . . . . . . . . 160

12. Rawls . . . . . . . . . . . . . 179

B.3 Schwach normative und

kontextualistische Ansätze . . . . . . . . . 191

Einleitung ................ 191

13. Kohärentismus . . . . . . . . . . . . . . . . . . . . . . . . . . .

14. Klugheitsethik . . . . . . . . . . . . 206

15. Kommunitarismus . . . . . . . . . . 218

16. Hermeneutische Ethik / Narrative Ethik . . 231

\section{Angewandte oder}

Bereichsspezifische Ethik . . . . . . 243

Einleitung . . . . . . . . . . 243

1. Bioethik . . . . . . . . . . 247

2. Genethik . . . . . . . . . . . . . 253

3. Kulturethik . . . . . . . . . . . 258

4. Medienethik . . . . . . . . . . . . 263

5. Medizinethik . . . . . . . . . . 268

6. Politische Ethik . . . . . . . . . . . 273
7. Technikethik . . . . . . . . . . . . 277

8. Tierethik . . . . . . . . . . . . 282

9. Umweltethik . . . . . . . . . . . . . . . . . 286

10. Wirtschaftsethik . . . . . . . . . . 291

IV. Zentrale Begriffe der Ethik . . . . . 297

Anerkennung . . . . . . . . . . . . . 297

Anthropologie . . . . . . . . . . . . 301

Autonomie . . . . . . . . . . . . . 305

Bedürfnisse / Interessen . . . . . . . . . . 308

Begründung . . . . . . . . . . 313

Deontische Logik . . . . . . . . . . . . 319

Dilemma . . . . . . . . . . . . . . . . 325

Entscheidungstheorie / Spieltheorie . . . . . 331

Erfahrung . . . . . . . . . . 336

Evolutionäre Ethik . . . . . . . . . . . . . 341

Feministische Ethik . . . . . . . . . . 346

Freiheit . . . . . . . . . . . . . 352

Gefühl / moral sense . . . . . . . . 360

Gerechtigkeit . . . . . . . . . . . . . 365

Gewissen . . . . . . . . . . . . . 371

Glück / Wohlergehen . . . . . . . . . . . 375

gut / das Gute / das Böse . . . . . . . . . . 381

Güterabwägung . . . . . . . . . . . . . 385

Handlung . . . . . . . . . . . . . 390

Identität . . . . . . . . . . . . . . . . 394

Kompromiss . . . . . . . . . . . . . . . 399

Konsens . . . . . . . . . . . . . 404

Moral . . . . . . . . . . . . 4 410

Moral point of view . . . . . . . . . 414

Moralischer Status . . . . . . . . . . . 417

Moralpädagogik . . . . . . . . . . . . 423

Moralpsychologie / Moralentwicklung . . . . 4428

Motivation . . . . . . . . . . . . 432

Naturalistischer Fehlschluss . . . . . . . . 437

Person . . . . . . . . . . . . . . 4440

Pflicht . . . . . . . . . . . . . . 447

Präferenzen . . . . . . . . . . . . . 453

Prinzip / Maxime / Norm / Regel . . . . . . . . 457

Rationalität . . . . . . . . . . . . . 463

Recht und Moral . . . . . . . . . . . . . . 469

Rechte . . . . . . . . . . . . 477 


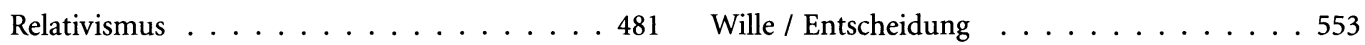

Risiko . . . . . . . . . . . . 485 Würde . . . . . . . . . . . 537

Sittlichkeit / Ethos . . . . . . . . . . 491

Sollen . . . . . . . . . . . . . . 496

Sozialethik . . . . . . . . . . . . 500

Theologie und Ethik (kath. Sicht) . . . . . . 504

Theologie und Ethik (protest. Sicht) . . . . . . 508

Tugend . . . . . . . . . . . . . . . 513

Universalisierung . . . . . . . . . 517

Verantwortung . . . . . . . . . . . 5 521

Wert . . . . . . . . . . . . . 527

Zweck / Ziel . . . . . . . . . . . . . 542

V. Anhang . . . . . . . . . . 547

1. Bibliographie . . . . . . . . . . . . . 547

2. Die Autorinnen und Autoren . . . . . . . . . . 551

3. Personenregister . . . . . . . . . . . 554

4. Sachregister . . . . . . . . . 568 


\section{Vorwort}

In kaum einer philosophischen Disziplin vollziehen sich derzeit so viele Veränderungen wie in der Ethik. Darüber hinaus ist Ethik inzwischen in ganz unterschiedlichen institutionellen Zusammenhängen $\mathrm{zu}$ finden. Neben den klassischen Orten ethischer Reflexion in Philosophie und Theologie wird Ethik derzeit in verschiedenen universitären Ausbildungen, in Fachhochschulen, in Akademien und Schulen etabliert. Themen der Angewandten Ethik sind häufig auch in öffentlichen und politischen Debatten präsent. Dabei ist eine zunehmende Auffächerung zwischen philosophischem Grundlagendiskurs und konkreten moralischen Orientierungsfragen zu beobachten. Die schnelle Entwicklung der Diskussion und die Ausdifferenzierung der ethischen Debatten macht den Zugang zu ethischen Fragen immer schwieriger. Das mit dem vorliegenden Handbuch verfolgte Vorhaben, Leserinnen und Lesern eine schnelle und gezielte, zugleich aber auch fachlich fundierte Orientierung im ethischen Diskurs zu bieten, scheint daher dringend notwendig. Aus genau denselben Gründen stellt es aber auch eine ernste Herausforderung dar.

Primäres Anliegen des Buches ist die Erschließung der aktuellen ethischen Fachdiskussion. Philosophiegeschichtliche Aspekte treten demgemäß eher in den Hintergrund. Freilich wird auf traditionelle Ansätze überall dort Bezug genommen, wo diese auch heute noch wichtige Beiträge zur Klärung von Sachproblemen liefern oder wo Begrifflichkeiten und Diskussionszusammenhänge ohne historisches Hintergrundwissen unverständlich bleiben müssten.

Das Handbuch ist von dem Versuch geprägt, metaund fundamentalethische sowie anwendungsethische Fragen gleichermaßen zu berücksichtigen. Dies ist im gegenwärtigen Ethik-Diskurs nicht mehr selbstverständlich. Durch die hier getroffene thematische Auswahl möchten wir einerseits der wachsenden Bedeutung von bereichsethischen Problemstellungen Rechnung tragen. Andererseits möchten wir deutlich machen, dass die Angewandte Ethik der Sache nach immer an den fundamental- und metaethischen Grundlagendiskurs zurückgebunden bleibt und dass Entwicklungen im einen Bereich stets für neue Herausforderungen im jeweils anderen sorgen.
Schließlich zeichnet sich das Handbuch durch eine neuartige Gliederung des Stoffs aus, indem es systematische mit lexikalischen Ordnungsgesichtspunkten kombiniert. Auf diese Weise werden Querverbindungen und Verweiszusammenhänge in den aktuellen Debatten, aber auch Gewichtungen und Rangunterschiede innerhalb der ethischen Begrifflichkeit erkennbar. Während etwa die Bezeichnungen ,Utilitarismus`, ,Diskursethikı oder 'Kommunitarismus` für umfassende ethische Theoriefamilien stehen, die von verschiedenen Autorinnen und Autoren in zum Teil ganz unterschiedlichen Spielarten vertreten werden, handelt es sich bei >Autonomier, 'Norm` oder 'Verantwortung begriffe, die in jeder der genannten Theorien eine mehr oder weniger bedeutsame Rolle spielen, dort jedoch wiederum ganz unterschiedlich interpretiert werden können. Um nun einerseits die charakteristische Eigenständigkeit umfassender ethischer Theorieansätze sowie ihre Zugehörigkeit zu bestimmten Theoriegruppen zum Ausdruck zu bringen, andererseits aber auch die im Hinblick auf diese Theorien vorliegende $>$ Mehrfachaffinität $<$ ethisch relevanter Begriffe deutlich zu machen, haben wir uns dafür entschieden, die Darstellung von Theorien und ethischen Zentralbegriffen sozusagen einander gegenüberzustellen.

Den drei systematischen Hauptteilen des Handbuchs geht eine umfangreiche Einleitung voran, die das Themenfeld in begrifflicher, historischer, metaethischer und zeitdiagnostischer Perspektive erkundet.

Im systematischen Teil des Handbuchs (Abschnitt II: Ethische Theorien im Überblick) werden dann verschiedene Gruppen ethischer Theoriebildung vorgestellt. Auf einen einführenden Abschnitt, der in drei Beiträgen die Grundprobleme der 'Metaethik sowie zentrale metaethische Diskussionen zur Sprache bringt, folgen drei Gruppen von jeweils verwandten normativen Theorien, und zwar: >Teleologische Ansätze`, ’Deontologische Ansätze` sowie schwach normative und kontextualistische Theorien`. Jeder der drei Abschnitte wird durch eine 
Einführung der Herausgeber eröffnet, in der vor allem auf die charakteristische Zusammengehörigkeit der vorgestellten Ansätze eingegangen wird. Der Anspruch dieses Teiles besteht somit darin, Leserinnen und Lesern eine Art $>$ Landkarter der gegenwärtigen fundamental- und metaethischen Diskussion an die Hand zu geben.

Der zweite Teil des Handbuchs (Abschnitt III: Angewandte oder Bereichsspezifische Ethik) ist der Angewandten Ethik gewidmet. In zehn Beiträgen werden die derzeit prominentesten Bereichsethiken vorgestellt, wobei auch diesem Teil eine Einführung der Herausgeber vorangeht. In ihr wird vor allem das schwierig zu bestimmende Verhältnis von Grundlagen- und Anwendungsdimension in der Ethik beleuchtet, so dass auch hier noch einmal - auf dem Niveau einer allgemeinen theoretischen Reflexion einsichtig gemacht wird, warum die nachfolgenden Einzelbeiträge immer wieder auf fundamentalethische Positionen Bezug nehmen.

Der dritte Teil (Abschnitt IV: Zentrale Begriffe der Ethik) präsentiert schließlich in lexikalischer Anordnung fünfzig Beiträge zu ethisch relevanten Grundbegriffen. Da diese Begriffe in den verschiedenen ethischen Ansätzen - und teilweise auch in den Bereichsethiken - nicht immer univok gebraucht werden, werden hier vor allem die Kerngehalte sowie die jeweilige Affinität zu unterschiedlichen ethischen Theorien aufgeschlüsselt. Ebenso soll deutlich werden, in welchen bereichsethischen Kontexten und in welchen Problemzusammenhängen diese Begriffe hauptsächlich Verwendung finden. Dass sich solche Begriffsklärungen von Fall zu Fall unterschiedlicher Zugangsweisen bedienen müssen - historisch oder systematisch-, versteht sich von selbst. Ebenso dürfte es auf der Hand liegen, dass eine notgedrungen begrenzte Auswahl von ethisch relevanten Grundbegriffen immer anfechtbar bleibt.

Ein allgemeines Literaturverzeichnis am Ende des Bandes soll einen raschen Zugriff auf Nachschlagewerke, Einführungsliteratur und besonders wichtige
Werke der Ethik ermöglichen. Neben einem Personenregister findet sich auch ein Sachregister, das die Möglichkeiten einer gezielten thematischen Suche im Handbuch noch einmal erweitert. Hier wird man auf einschlägige Passagen zu Stichworten verwiesen, denen aus Platzgründen kein eigener Artikel gewidmet werden konnte.

Unter den Autorinnen und Autoren des Handbuchs finden sich Vertreter/innen ganz verschiedener Denkrichtungen. Auch die Herausgeber fühlen sich unterschiedlichen Ethikansätzen verpflichtet. Dementsprechend differieren die einzelnen Beiträge in ihren Bewertungen und Gewichtungen, ebenso ist die Einheitlichkeit in der Begriffsverwendung und im Verständnis metaethischer Systematisierungen nicht durchgehend garantiert. Zur Vermeidung von Missverständnissen haben wir in einigen wenigen Fällen auf Differenzen aufmerksam gemacht. In jedem Fall aber haben sich Autorinnen und Autoren bemüht, theoretische Vorannahmen und Begriffe sorgfältig einzuführen. Insofern hoffen wir, dass dieses Handbuch bei dem Versuch hilfreich ist, sich im ethischen Denken der Gegenwart zu orientieren, und dass zugleich etwas von der Vielfalt der gegenwärtigen ethischen Debatten deutlich wird.

Wir danken allen Autorinnen und Autoren für ihre Bereitschaft zur Mitwirkung und für die freundliche Zusammenarbeit. Obwohl jeder der drei Herausgeber während der Erstellung des Handbuchs seinen institutionellen Kontext gewechselt hat, gelang die Kooperation erstaunlich gut. Ute Hechtfischer vom Metzler-Verlag hat das Entstehen dieses Handbuchs angeregt, alle Phasen seiner Entwicklung konstruktiv begleitet und Verzögerungen und technische Probleme mit einer vorbildlichen Mischung aus freundlicher Ermahnung, Ermutigung und Geduld ertragen. Ihr danken wir ganz herzlich.

Utrecht, Nijmegen und Freiburg im Sommer 2002

M. Düwell, Ch. Hübenthal und M.H. Werner 